\title{
Mitteilungen
}

\section{Erkki Itkonen 1913-1992}

Erkki Itkonen, Mitglied der Akademie Finnlands, Prof. emerit. für finnischugrische Sprachforschung an der Universität Helsinki, ist am 28.5.1992 in Helsinki verstorben.

Erkki Itkonen wurde am 26. April 1913 in Inari als jüngster Sohn des Pfarrers Lauri Itkonen geboren. Er stammte aus einer Familie, deren Verdienste um die Erforschung der lappischen Sprache und Kultur wie auch um die praktische Förderung der Lappen und ihrer Kultur in Finnland unvergleichlich groß sind. Aufgewachsen ist Erkki Itkonen allerdings in Südfinnland, da sein Vater schon ein Jahr nach der Geburt des jüngsten Kindes die Gemeinde Vihti als Pfarrherr übernahm. Einer der älteren Brüder von Erkki Itkonen wurde jedoch selbst Pfarrherr von Inari; damals war Erkki Itkonen erst zehn Jahre alt. Die Verbindungen der Familie zum hohen Norden blieben also erhalten.

Das Studium absolvierte Erkki Itkonen an der Universität Helsinki, wo er bereits mit zwanzig Jahren die Magisterprüfung ablegte und sechs Jahre später, 1939, zum Dr. phil. promovierte. Der junge Erkki Itkonen, der bereits im Alter von zwanzig Jahren seine erste Untersuchung über ein etymologisches Thema veröffentlicht hatte, fühlte sich von zwei wissenschaftlichen Disziplinen besonders angezogen, der Folkloristik und der Sprachwissenschaft. Auf einer Reise nach Inari im Sommer hat er sich dann für die Sprachwissenschaft entschieden, obwohl sein Interesse für die Folkloristik, vor allem für die geistige Überlieferung der Lappen, immer lebendig geblieben ist und er später auch entsprechende beeindruckende Forschungsarbeiten vorgelegt hat.

In seiner Dissertation "Der ostlappische Vokalismus vom qualitativen Standpunkt aus mit besonderer Berücksichtigung des Inari- und Skoltlappi- 
schen. Lautgeschichtliche Untersuchung." MSFOu 79. XVI + 386 S. (1939) zeigte der junge Verfasser bei der Behandlung des anspruchsvollen Themas bereits seine besonderen Fähigkeiten. Die selbstverständliche Berücksichtigung des Systemganzen, wie sie auch für die späteren Arbeiten von Itkonen charakteristisch ist, kommt bereits hier in der Fähigkeit, ein großes, repräsentatives Material ganzheitlich zu behandeln, zum Ausdruck. Während seine akademischen Lehrer die junggrammatische Schule vertraten, machte sich der junge Forscher auf eigene Faust mit der in Mitteleuropa aufgekommenen Phonologie vertraut. Die Klärung der qualitativen Struktur des komplizierten ostlappischen Vokalismus, der zahlreiche Umlautwechsel aufweist, und die Analyse der Entwicklung, die zu dieser Struktur geführt hat, kann als erster großer Gewinn für den Forscher Erkki Itkonen bezeichnet werden; sieben Jahre später führte er die Arbeit weiter, indem er sich nun auf das ostlappische Quantitätssystem konzentrierte. Es entstand die Arbeit "Struktur und Entwicklung der ostlappischen Quantitätssysteme" (MSFOu 88. XXII + 267 S.), wo die außerordentlich differenzierten, fast chaotisch wirkenden Quantitätswechsel als ein überschaubarer Kosmos dargestellt werden. Neben diesen großen Monographien entstanden kleinere Arbeiten über Grundfragen der lappischen Lautlehre "“̈̈ber den Charakter des ostlappischen Stufenwechselsystems", FUF 27 1941; "Die Stammbildung bei den lappischen $a$-Stämmen" a. a. O.; "Varhaiskantasuomen $a: n$ ja $\ddot{a}: n$ kehitys lapissa" - Referat: Die Entwicklung der frühurfinnischen $a$ und $\ddot{a}$ im Lappischen. Vir. 1950). Mit Problemen der lappischen Lautlehre befaßte sich Itkonen auch später $z$. B. in Vorträgen, die er als Vorsitzender der Finnisch-Ugrischen Gesellschaft bei den jeweiligen Jahresversammlungen hielt ("Phonetische und phonologische Betrachtung der ostlappischen Dialekte", JSFOu 72 1973; "Betrachtungen zum lappischen Stufenwechsel", JSFOu 75 1977). Seine grundlegende Kenntnis der Phonologie der lappischen Sprachen konnte Itkonen auch bei der Entwicklung der Orthographie für die fjäll-lappische Schriftsprache nutzen; dieser Arbeit opferte er viel Zeit. Auch für das phonematische Schriftsystem des Inarilappischen und Kildinlappischen (Šonguj-Dialekt) hat er Vorschläge ausgearbeitet (Castrenianumin toimitteita 1, 1971).

Die durch Itkonen erfolgte Klärung der lappischen Lautlehre warf neues Licht auf die frühen Phasen der finnischen Sprache. Neue, tiefschürfende Deutungen erfuhren das Vokalsystem des Urfinnischen und des Frühurfinnischen wie auch die Morphophonologie des Finnischen und Erscheinungen der Stammtypen des Finnischen ("Varhaiskantasuomen pääpainottoman ai-diftongin kahtalaisen kehityksen syistä" - Referat: Über die Ursachen der doppelten Entwicklung des frühurfinnischen nichthaupttonigen ai-Diphtongs. Vir. 1942; "Onko kantasuomessa ollut keskivokaaleja?" - Referat: Hat es im Urfinnischen mittlere Vokale gegeben? Vir. 1945; "Vokaalikombinaatiot ja vartalotyypit" - Referat: Vokalkombinationen und Stammtypen. Vir. 1948; "Beiträge 
zur Geschichte der einsilbigen Wortstämme im Finnischen", FUF 30 1949; "Über die suffixalen Labialvokale im Lappischen und Ostseefinnischen", Scandinavica et Fenno-Ugrica 1954; "Die Umwandlung einiger $a$ - und $\ddot{a}$ Stämme zu $e$-Stämmen im Urfinnischen”, JSFOu 75 1977). Das Vokalsystem der dem Frühurfinnischen vorangegangenen Stadien der Sprache beschäftigte ihn ebenfalls. So entstand seine klassische Arbeit "Zur Frage nach der Entwicklung des Vokalismus der ersten Silbe in den finnisch-ugrischen Sprachen, insbesondere im Mordwinischen" (FUF 29 1946). Die Besonderheit des Moksamordwinischen, die reduzierten Vokale, wurden später noch einmal ausführlich analysiert: "Zum Ursprung und Wesen der reduzierten Vokale im Mordwinischen" (FUF 39 1971). Die Fragen des Vokalismus der permischen Sprachen erhielten bereits 1951 eine Lösung, als Itkonen in der Zeitschrift Virittäjä seine Untersuchung "Permiläisen vokaali- ja painotusopin alalta" (Referat: Aus der permischen Vokal- und Akzentuationslehre) veröffentlichte. Eine umfangreiche Beschreibung der Geschichte des Vokalismus sowohl der permischen Sprachen als auch des Tscheremissischen erschien im Jahre 1954: "Zur Geschichte des Vokalismus der ersten Silbe im Tscheremissischen und in den permischen Sprachen" (FUF 31). In jenen Nachkriegsjahren, als es praktisch keinen Kontakt gab zwischen den finnischen und sowjetischen Wissenschaftlern, hatten Itkonen in Finnland und V. I. Lytkin in der Sowjetunion unabhängig voneinander die Probleme der Geschichte des permischen Vokalismus geklärt. In den wesentlichen Punkten waren beide zu denselben Resultaten gekommen. Itkonen hat durch seine Darstellung der Entwicklung des Vokalismus im Tscheremissischen endgültig die Grundlage der Vokaltheorie von Steinitz erschüttert, indem er nachweisen konnte, daß die reduzierten Vokale des Tscheremissischen aus der Zeit der Sonderentwicklung des Tscheremissischen stammen, aus dem Urtscheremissischen. Auch später schrieb Itkonen wichtige Arbeiten über Sonderfragen des Vokalismus in den permischen Sprachen: "A propos des voyelles réduites non arrondies de la première syllabe en tchérémisse" (Études Finno-Ougriennes 8 1972). Die Beschäftigung mit dem Vokalismus dieser Sprachen führte ihn auch in den Bereich der Turkologie; so entstand die Arbeit "Bemerkungen über den Vokalismus der ersten Silbe von tschuwaschischen Lehnwörtern in einigen finnischugrischen Sprachen" (FUF 38 1970). Itkonen veröffentlichte auch umfangreiche Synthesen über seine Untersuchungen zum Vokalismus und generell zur Lautlehre: "Thesen und Antithesen in der finnisch-ugrischen Vokalforschung" (UAJb 41 1969); "Zur Wertung der finnisch-ugrischen Lautforschung" (a. a. O.).

Itkonens Forscherinteresse galt allen Bereichen der Sprache. Seine Untersuchungen über die Morphologie enthalten eine erstaunliche Menge Wissen. Man erkennt in diesen Arbeiten seine außerordentlich gründliche Beherrschung der verschiedenen finnisch-ugrischen Sprachen. Hierher gehört vor allem die Arbeit "Onko itämerensuomessa jälkiä duaalista?" - Referat: Gibt es 
im Ostseefinnischen Spuren des Duals? (Vir. 1955), wo Itkonen das in dieser sprachlichen Besonderheit früher gesehene Hindernis der FrühurfinnischHypothese ausräumt; erwähnenswert sind auch in dem Zusammenhang seine "Beobachtungen über die Entwicklung des tscheremissischen Konjugationssystems" (MSFOu 125 1962), die eine neue Gesamtlösung für die Probleme der tscheremissischen Konjugation brachten. Wesentlich und inhaltsreich sind auch folgende Untersuchungen von ihm: "Zur Frühgeschichte der lappischen und finnischen Lokalkasus" (CIFU 2 1968), "Kuolanlapin vokatiivimuodoista" - Referat: Über die Vokativformen im Kolalappischen (Vir. 1947); "Das Perfekt des Partizips im Lappischen" (MSFOu 98 1950); "Über den Genetiv und Partitiv in einigen Adverbien" (CIFU 1 1963); "Die lappischen Deminutivableitungen von Superlativen und Komparativen, zunächst im Lichte des Inarilappischen" (Studia Ethnographica Upsaliensia 21, 1964); "Über einige Formen der dritten Person in der lappischen Konjugation" (FUF 37 1969); "Über einige lappische Verbalsuffixe" (JSFOu 76 1980); "TŠeremissin kielen sanaliittojen suhteesta yhdyssanoihin ja taivutusmuotoihin" - Referat: Über das Verhältnis von Wortverbindungen zu Komposita und Flexionsformen im Tscheremissischen (Vir. 1960); "Eräästä ťeremissin adverbityypistä" - Referat: Über einen tscheremissischen Adverbtyp (Vir. 1966); "Eräiden latiivin ja prolatiivin päätteiden tarkastelua" - Referat: Einige Lativ- und Prolativendungen (Vir. 1967); "Esiintyykö $s$-latiivi permiläisissä kielissä?" - Referat: Kommt der $s$ Lativ in den permischen Sprachen vor? (Vir. 1966). Eine aufschlußreiche Gesamtbetrachtung der finnisch-ugrischen Laut- und Formenlehre ist enthalten in dem Aufsatz "Die Laut- und Formenstruktur der finnisch-ugrischen Grundsprache" (UAJb 34 1962).

Schon als junger Lappologe hatte sich Itkonen auch für die Syntax begeistert: "Satakuntalaista lainaperua ruotsinlapin syntaksissa" - Referat: Lehnerbe der Satakunta-Mundart in der Syntax des Schwedischlappischen (Vir. 1938). Auch in seinen morphologischen Untersuchungen berücksichtigte Itkonen stets das Verhältnis zwischen Form und Funktion; vor allem zu Beginn der 70er Jahre scheint ihn die historische Syntax besonders interessiert zu haben. Davon zeugen zwei umfangreiche, inhaltsreiche Arbeiten: "Über das Objekt in den finnisch-wolgaischen Sprachen" (FUF 39 1972); "Zur Geschichte des Partitivs" (FUF 40 1973).

In Itkonens diachroner Forschung war auch Platz für die Synchronie. Die Geschichte der Sprache war für ihn die Realität der jeweiligen sprachlichen Gesamtheit. In all seinen historischen Abhandlungen strebt er immer wieder eine dem jeweiligen Zeitpunkt entsprechende synchrone Systemgesamtheit an. Es war für ihn eine Selbstverständlichkeit, daß er die Entwicklung der allgemeinen Sprachwissenschaft verfolgte. Auch seinen Schülern gegenüber betonte er die Wichtigkeit der allgemeinen Sprachwissenschaft und des Studiums der Erforschung anderer Sprachbereiche. Auch der typologische Aspekt in- 
teressierte ihn; zur Erweiterung seines eigenen Horizontes machte er sich beispielsweise mit den Sprachen im Bereich des Pazifik vertraut. Für sein allgemeinsprachwissenschaftliches Werk "Kieli ja sen tutkimus" (Die Sprache und ihre Erforschung; 1966) nahm er das Beispielmaterial jedoch aus seiner souveränen Kenntnis der finnisch-ugrischen Sprachen.

Mit zwanzig Jahren hatte Itkonen seine Karriere mit einer wortgeschichtlichen Untersuchung begonnen. Auch wenn er später in die hehren Bereiche der autonomen Sprachwissenschaft vorstieß, hat er doch nie die Erdverbundenheit aus den Augen verloren, die der Kontext bedeutet, in dem die Sprache lebt. Das Wissen um das Leben der Sprache setzte für ihn auch Kenntnisse voraus in der Archäologie, der Geschichte, der Ethnographie, der Folklore, der Literatur. In seinen wortgeschichtlichen Abhandlungen über das Lappische führte er den Leser in weite Vergangenheiten zurück, die er lebendig zu machen vermochte: "Lappalaista merkitsevistä nimityksistä ja niiden historiallisesta taustasta" - Résumé: Les termes désignant les Lapons et leur fond historique (Vir. 1941); "Lappalaisten esihistoriaa valaisevia sanoja" - Résumé: Mots éclairant le préhistoire des Lapons (Vir. 1946); "Die Herkunft und Vorgeschichte der Lappen im Lichte der Sprachwissenschaft" (UAJb 27 1955); "Zwei Andenkeit von Karelien und Haalogaland im Lappischen" (MSFOu 145 1968); "Über die Etymologie von fi. mahla 'Baumsaft' und maaliskuu 'März"' (FUF 24 1937). Wichtige etymologische Arbeit leistete Itkonen auch im Zusammenhang mit der etymologischen Klärung des zu seinem lappischen Lehrbuch gehörenden Teils über die Lexik: "Lappische Chrestomathie mit grammatikalischem Abriss und Wörterverzeichnis" (1960). Den größten Dienst hat Itkonen der Erforschung des lappischen Wortschatzes jedoch mit seinem umfangreichen Lexikon "Inarilappisches Wörterbuch" 1-4 (1986-1991) geleistet. Itkonen hatte einen wirklichen Thesaurus des Inarilappischen angestrebt, der alle Angaben über diese kleine vom Untergang bedrohte Sprache enthalten sollte; ein solches Wörterbuch hat er auch geschaffen. Das auf den 1331 Seiten mitgeteilte lexikalische Material zeichnet sich durch besonders vielseitige Beispiele aus. Als Band 4 des Wörterbuchs sind Itkonens grammatikalische Aufzeichnungen über das Inarilappische erschienen. Zum überwiegenden Teil beruht das Wörterbuch auf dem von Itkonen selbst im Terrain gesammelten Material. Jeweils im Sommer betrieb er Feldforschung. Mit besonderer Wärme erinnerte er sich später an die Sommer in einem Einödhaus im östlichen Inari-Gebiet, wo er seinen treuen Freund, den Inari-Lappen Uula Morottaja, interviewte. Hoffentlich werden auch die Textproben, die Itkonen von Uula Morottaja aufgezeichnet hat, in Zukunft veröffentlicht werden. Die Texte, die Itkonen aus einer anderen ostlappischen Sprachform, dem Kildinlappischen, gesammelt hat, sind bereits publiziert: "Kildinlappische Sprachproben" (MSFOu 191 1985).

Für die Wortgeschichte seiner Muttersprache hat Itkonen eine Arbeit geleistet, die allein schon ausgereicht hätte, ihn zu der überragenden Gestalt zu 
machen, als die er in die Geschichte der Finnougristik eingegangen ist. Nach dem Tod von Y. H. Toivonen übernahm Erkki Itkonen die Verantwortung für die Fertigstellung des etymologischen Wörterbuches der finnischen Sprache, wobei er auf viele eigene Forschungspläne verzichten mußte, was ihm nicht immer leicht gefallen ist. Er warb dann Aulis J. Joki und Reino Peltola als Mitarbeiter, und gemeinsam sorgten sie dafür, daß das Finnische Etymologische Wörterbuch fertiggestellt wurde; Band 6, der letzte Teil, erschien 1978. (Es folgte noch Band 7, der Register-Teil.) Dieses monumentale Werk, das seinen Verfassern zur Ehre gereicht, erhält derzeit einen Ableger, ein Lexikon, das für das große Publikum gemeint ist ("Suomen sanojen alkuperä" [Die Herkunft der finnischen Wörter]); es entsteht im Forschungszentrum für die Landessprachen Finnlands und Erkki Itkonen hat noch in seinen letzten Tagen am Manuskript gearbeitet. Was der finnische Wortschatz über die Geschichte der Sprache aussagen kann, darüber hat Itkonen z. B. in seinem Aufsatz "Die Vorgeschichte der Finnen aus der Perspektive eines Linguisten" (UAJb 32 1960) geschrieben. Ein Verdienst von Erkki Itkonen im Zusammenhang mit der Erforschung der Lexik wird erst in der Zukunft sichtbar werden: Auch aus dem Tscheremissischen hat er mit der ihm eigenen Sorgfalt und Einfühlung Wortschatzmaterial aufgezeichnet, das enthalten sein wird in dem Tscheremissischen Wörterbuch, das im Forschungszentrum für die Landessprachen Finnlands erstellt wird.

Erkki Itkonen, der in seiner Jugend zwischen Folkloristik und Sprachwissenschaft schwankte, hat die Volksdichtung und die Dichtung überhaupt auch später nie aus den Augen verloren. Vor allem die Volksdichtung der Lappen und überhaupt ihre geistige Überlieferung hat ihn zu weiterführenden, ergebnisreichen Untersuchungen angeregt: "Lappalainen kansanrunous" (Die lappische Volksdichtung) (in: Suomen kirjallisuus I 1963), "Kolttien satu revontulien synnystä" - Referat: Das Märchen vom Ursprung der Nordlichter bei den Skoltlappen (Vir. 1959); "Lappalainen haltiatarpari ja sen siperialainen vastine" (Zwei lappische feminine Geister und ihre sibirischen Entsprechungen; KV 40 1960); "Hajahuomioita lappalaisten perinteestä" (Einige Beobachtungen zur lappischen Überlieferung; KV 54 1974); “Älteste Elemente der lappischen Volksüberlieferung" (JSFOu 74 1976); "Olaus Sirman kotiseudusta ja kielestä" - Referat: Über die Heimat und die Sprache von Olaus Sirma (Vir. 1940); "Suomenlappalaisia kirjailijoita" (Finnisch-lappische Schriftsteller; KV 51 1971).

Schon zu Lebzeiten hat Erkki Itkonen zahlreiche Anerkennungen und Ehrungen erfahren. Die Universitäten Uppsala, Turku und Debrecen haben ihn zum Ehrendoktor ernannt, die Ungarische Akademie der Wissenschaften hat ihn zum Ehrenmitglied berufen, zahlreiche einheimische und ausländische wissenschaftliche Gesellschaften haben ihn zu ihrem Ehrenmitglied gewählt. Er hatte wichtige Vertrauensposten inne: Bis zum Jahre 1978 fungierte er als 
Vorsitzender der Finnisch-Ugrischen Gesellschaft, nachdem er zuerst als junger Mann lange Zeit Sekretär und danach stellvertretender Vorsitzender der Gesellschaft gewesen war. In der Societas Uralo-Altaica wirkte er lange Jahre als Vizepräsident. Lange Zeit hat er auch in der Lapin sivistysseura (Lappische Kulturgesellschaft) gewirkt, als Vorsitzender bis 1971; 22 Jahre lang hat er die lappische Zeitung der Gesellschaft, Sapmelaš, herausgegeben und weitgehend mit Beiträgen beliefert. Auch die Herausgabe zahlreicher wissenschaftlicher Publikationen und Serien lag in seinen Händen. Mehr als dreißig Jahre lang wirkte er unermüdlich als Chefredakteur der Finnisch-Ugrischen Forschungen.

Die ehrenvolle Anerkennung, die ihm in der Welt der Wissenschaft dank seiner wissenschaftlichen Verdienste zuteil wurde, begegnete ihm von seiten derer, denen es vergönnt war, in seiner Nähe zu arbeiten, auch aufgrund seiner Charaktereigenschaften. In ihm vereinten sich ein großer Wissenschaftler und ein großer Mensch. M. A. Castrén hat einmal von seinem intensiven Bedürfnis gesprochen, das eigene $\mathrm{Ma} B$ zu erfüllen - dieses Streben, verbunden mit einer tiefen Humanität, war auch kennzeichnend für Erkki Itkonen. 